\title{
Reflets
}

Revue ontaroise d'intervention sociale et communautaire

\section{Intervenir auprès des personnes ayant une perte auditive}

\section{Jean-Roger Brisebois}

Volume 4, numéro 2, automne 1998

Personnes vivant avec une incapacité

URI : https://id.erudit.org/iderudit/026225ar

DOI : https://doi.org/10.7202/026225ar

Aller au sommaire du numéro

Éditeur(s)

Reflets : Revue ontaroise d'intervention sociale et communautaire

ISSN

1203-4576 (imprimé)

1712-8498 (numérique)

Découvrir la revue

Citer cet article

Brisebois, J.-R. (1998). Intervenir auprès des personnes ayant une perte auditive. Reflets, 4(2), 151-154. https://doi.org/10.7202/026225ar

Tous droits réservés (C) Reflets : Revue ontaroise d'intervention sociale et communautaire, 1998
Ce document est protégé par la loi sur le droit d'auteur. L'utilisation des services d'Érudit (y compris la reproduction) est assujettie à sa politique d'utilisation que vous pouvez consulter en ligne.

https://apropos.erudit.org/fr/usagers/politique-dutilisation/ 


\title{
Intervenir auprès des personnes ayant une perte auditive
}

\author{
Jean-Roger Brisebois \\ Travailleur social, Sudbury
}

Il y a quelques mois, on m'a demandé de décrire brièvement mon expérience de travail auprès des personnes ayant une perte auditive. Dans cet article, en plus d'aborder l'accueil par l'intervenant et le genre d'intervention pratiquée, je présenterai les services offerts aux personnes sourdes ou devenues sourdes et malentendantes, afin d'informer et d'aider ceux qui travaillent ou travailleront auprès de cette clientèle.

Je suis malentendant, c'est-à-dire que j'ai une perte auditive. Selon les examens auditifs que je reçois annuellement depuis mon enfance, ma perte est jugée «modérée». Médicalement, il n'y a aucune intervention chirurgicale qui puisse être pratiquée, car c'est une perte neurosensorielle. Je porte deux prothèses auditives qui jouent le rôle d'amplification, pour m'aider à entendre. En d'autres mots, c'est comme un système de son où l'on augmente le volume jusqu'à ce que le son soit clair. Il est à noter que tous les bruits environnants sont aussi amplifiés, ce qui rend parfois de très simples conversations difficiles à entendre.

Beaucoup de gens font la comparaison entre les prothèses auditives et les verres optiques. Lorsque nous portons des lunettes, notre vision devient presque parfaite. Dans le cas des prothèses auditives, ce n'est malheureusement pas le cas. Les prothèses auditives aident seulement à entendre des sons à un volume plus élevé. En réalité, une grande majorité de malentendants et de sourds sont incapables de porter des prothèses auditives dans les endroits où il y a beaucoup de bruit. C'est comme écouter un 
poste de radio lorsque la réception est mauvaise ou lorsqu'il y a beaucoup d'interférences.

Depuis neuf mois, je travaille comme conseiller à la Société canadienne de l'Ouïe (SCO) à Sudbury. On m'a embauché spécifiquement pour desservir la clientèle sourde en santé mentale. La société espère mettre sur pied un service en santé mentale pour les personnes sourdes ou devenues sourdes et malentendantes. En effet, plusieurs recherches ont démontré que ce groupe cible ne reçoit pas des services adéquats en ce domaine. En décembre 1997, la SCO a soumis une proposition au Ministère de la santé, des services sociaux et communautaires afin de pouvoir offrir ce service spécialisé en santé mentale. Récemment, le gouvernement a annoncé qu'il ne peut pas supporter un tel projet, car il existe déjà un organisme de santé mentale pour desservir cette clientèle.

À Sudbury, nous avons un excellent service de santé mentale. Le problème, c'est qu'il n'est pas tout à fait sensibilisé aux besoins de la clientèle des personnes sourdes ou devenues sourdes et malentendantes. Ainsi, leurs évaluations de cette clientèle sont loin d'être toujours adéquates. Mon expérience me fait croire que le service de santé mentale tient pour acquis que cette clientèle peut communiquer de façon orale, ce qui est loin d'être toujours le cas. Mon intention n'est pas de critiquer le service en question, je l'ai dit, il est excellent. Toutefois, j'aimerais simplement faire quelques suggestions pour améliorer le service. Ces suggestions, tirées de ma pratique à la SCO, peuvent s'avérer toutes aussi bénéfiques pour n'importe quelle agence.

Lors de l'accueil, il faut demander à la personne si elle a une perte auditive. Par expérience, nous savons très bien que si nous ne posons pas de questions, les gens n'aborderont pas le sujet d'eux-mêmes. Il faut aussi demander à la personne si elle s'identifie comme personne sourde ou devenue sourde et malentendante. Cette identification rendra notre tâche plus facile. Cela dit, la plupart du temps, une personne niera qu'elle a une perte auditive. Il faut donc observer attentivement la manière dont la personne communique. Parle-t-elle fort? A-t-elle bien compris la question? Nous demande-t-elle de répéter souvent? 
Il est primordial d'évaluer, si oui ou non la personne a une perte auditive avant de passer à l'intervention. La perte auditive est un handicap invisible. Et si ces personnes n'avaient pas besoin de communiquer, nous ne pourrions même pas dire qu'elles sont «handicapées». C'est ce qui rend l'évaluation de la perte auditive si difficile. Un grand nombre d'entre elles nient qu'elles ont une perte auditive et réussissent à nous faire croire qu'elles entendent bien. C'est ce refus qui engendre le plus de difficultés. Peu importe le problème, qu'il soit relié au milieu de travail, conjugal ou familial, le refus de reconnaitre la perte auditive amplifie le problème. Ensuite, il faut évaluer si la source du problème est au niveau de la communication.

Il est toujours plus facile d'intervenir lorsqu'on connait la manière dont la personne communique. Évidemment, il est facile d'identifier une personne sourde puisqu'elle se sert de la langue des signes comme méthode de communication. Bien que la plupart des personnes sourdes ne parlent pas, elles émettent néanmoins des sons lorsqu'elles communiquent. Il est même possible de rencontrer des personnes sourdes qui maitrisent bien la langue parlée, soit en raison d'un entraînement oral rigoureux (méthode oraliste), soit que la surdité est survenue après l'acquisition du langage.

Plus que le simple repérage de la perte auditive lors de l'accueil, dont l'importance n'est plus à démontrer, il importe de vérifier également le niveau de compréhension du client. En effet, l'information n'est pas toujours bien reçue chez le client sourd ou malentendant. On doit donc s'assurer qu'il a bien compris. Pour ce faire, il y a différents moyens. Règle générale, les personnes ayant une perte auditive ne demandent pas de répéter et nous assurent avoir bien compris l'information du premier coup. Afin d'aider ces personnes, il faut donc insister pour qu'elles nous répètent l'information transmise en leurs propres mots. Comme ça, on est certain d'être sur la même longueur d'ondes.

Une fois la surdité assumée et la méthode de communication établie, il est maintenant possible de continuer le processus de l'intervention. Que la personne soit sourde ou devenue sourde et malentendante, les prochaines étapes de l'intervention 
demeurent les mêmes que celles effectuées avec une personne entendante.

À ce niveau, on utilise plutôt des approches holistiques et systémiques. Lors de l'accueil, c'est d'ailleurs à une approche holistique à laquelle nous avons eu recours pour montrer l'importance de bien comprendre tous les aspects liés à la façon dont le client donne et reçoit de l'information. Pendant l'intervention, on consacre beaucoup de temps à montrer au client à bien communiquer. Au fur et à mesure qu'on avance dans l'intervention, on se concentre sur les besoins particuliers du client. Nous évaluons la manière dont il se débrouille pour surmonter les défis de tous les jours. Si nécessaire, nous pouvons poursuivre notre évaluation et voir la façon dont le client interagit avec ses systèmes. Nous essayons de comprendre si ses systèmes de famille, d'école, d'amis ou de travail favorisent chez lui, l'atteinte d'une vie indépendante. L'intervention est surtout offerte à ceux qui ont de la difficulté à équilibrer leurs défis.

Pour ceux qui n'ont pas besoin d'intervention, la SCO offre une gamme de services spécialisés: services d'interprètes de langues signées en L.S.Q. (Langue Signée Québécoise) et en A.S.L. (American Sign Language), services d'emploi où l'on apprend à se préparer pour le milieu du travail, services d'alphabétisation pour les personnes ayant une surdité et, enfin, les services de ressources en surdité.

En conclusion, $\mathrm{j}$ 'aurais un message à adresser à tous les intervenants qui travaillent ou travailleront auprès de cette clientèle. La perte auditive est un handicap invisible qui n'est pas compris par la société et qui demande beaucoup d'attention de la part des intervenants. Alors, n'hésitez pas à communiquer avec la Société canadienne de l'Ouie pour de plus amples renseignements. Ces services spécialisés ont pour but ultime de procurer aux personnes sourdes, devenues sourdes et malentendantes, l'indépendance et l'autonomie nécessaires pour profiter pleinement de la vie. 